\title{
ITERATIVE CONTINUOUS COLLOCATION METHOD FOR SOLVING NONLINEAR VOLTERRA INTEGRO-DIFFERENTIAL EQUATIONS
}

\author{
AZZEDDINE BELLOUR AND KHAOULA ROUIBAH
}

\begin{abstract}
In this paper, an iterative collocation method is presented for a class of nonlinear Volterra integro-differential equations. This iterative collocation method is made by using a suitable continuous spline polynomials. It is shown that this method is convergent. The results are compared with the results obtained by other well-known numerical methods to prove the effectiveness of the presented algorithm.
\end{abstract}

\section{Introduction}

In this paper, we study a numerical method for the solution of the following nonlinear Volterra integro-differential equations,

$$
\begin{aligned}
& x^{\prime}(t)=f(t)+Q(t, x(t))+\int_{0}^{t} K\left(t, s, x(s), x^{\prime}(s)\right) d s, t \in I=[0, T] \\
& x\left(t_{0}\right)=x_{0}
\end{aligned}
$$

where the functions $f, Q, K$ are sufficiently smooth. The existence and the uniqueness of the solution of (1.1) can be found, for example, in [3]. The integrodifferential equations have become important in the mathematical modeling of many field of sciences and engineering (see, e.g., [3, 12, 17]).

Recently, many numerical methods have been proposed to approximate the solution of integro-differential equations. For example, Taylor collocation method, Runge-Kutta methods, collocation method, Legendre collocation method, Haar Wavelet method (cf, e.g. [1, 4, 5, 6, 8, 9, 10, 11, 13, 17]).

The aim of this paper is to generalize the iterative continuous collocation method in [14] for construct an iterative continuous approximate solution for equation (1.1). In our method the approximate solution is explicit, direct and obtained by using simple iterative formulas.

The main idea of the iterative collocation method is to obtain an explicit solution without needed to solve any algebraic system. Many authors used this method to solve the integral equations. L. Hacia [7], used the Iterative-Collocation Method to solve integral equations of heat conduction problems, H. Brunner [2] applied

2010 Mathematics Subject Classification. 45J05, 65R20.

Key words and phrases. Volterra integro-differential equations, Collocation method, Continuous spline polynomials, Iterative Method; Convergence analysis. 
the iterated collocation methods to approximate the solution for Volterra integral equations with delay arguments. In $[13,15]$ the variational iteration method is used to solve integral and integro-differential equations.

The outlines of this paper is as follows. In section 2, an iterative collocation method has been used to construct an approximate solution for (1.1) in the continuous spline polynomials space $S_{m}^{(0)}\left(\Pi_{N}\right)$, the convergence analysis has been given in section 3 . Some numerical illustrations are provided in section 4 .

\section{Description of the collocation method}

Let $\Pi_{N}$ be a uniform partition of the interval $I=[0, T]$ with grid points $t_{n}=n h, \quad n=0, \ldots, N-1$, where the stepsize is given by $h=\frac{T}{N}$. Let the collocation parameters be $0<c_{1}<\ldots \ldots<c_{m}<1$ and the collocation points $\Gamma_{N, m}=\left\{t_{n, j}=t_{n}+c_{j} h, j=1, \ldots, m, n=0, \ldots, N-1\right\}$. Define the subintervals $\sigma_{n}=\left[t_{n}, t_{n+1}\right]$, and $\sigma_{N-1}=\left[t_{N-1}, t_{N}\right]$.

Moreover, denote by $\pi_{m}$ the set of all real polynomials of degree not exceeding $m$.

We consider polynomial spline approximations $u(t)$ of the exact solution $x(t)$ in the spline space

$$
S_{m}^{(0)}\left(\Pi_{N}\right)=\left\{u \in C(\mathrm{I}, \mathbb{R}): u_{n}=u / \sigma_{n} \in \pi_{m}, n=0, \ldots, N-1,\right\} .
$$

This is the space of piecewise polynomials of degree at most $m$. Its dimension is $N m+1$, the same as the number of collocation points.

We seek $u \in S_{m}^{(0)}\left(\Pi_{N}\right)$ satisfies the collocation equation

$$
\begin{aligned}
& u^{\prime}(t)=f(t)+Q(t, u(t))+\int_{0}^{t} K\left(t, s, u(s), u^{\prime}(s)\right) d s, \quad t \in \Gamma_{N, m}, \\
& u\left(t_{0}\right)=f(0) .
\end{aligned}
$$

In what follows, we consider two equivalent reformulations of problem (2.1) by using the function $w(t)=u^{\prime}(t) \in S_{m-1}^{(-1)}\left(\Pi_{N}\right)$. Since $w_{n} \in \pi_{m-1}$, it holds for $\mu \in(0,1]$,

$$
\begin{gathered}
w_{n}\left(t_{n}+\mu h\right)=\sum_{v=1}^{m} L_{v}(s) w_{n}\left(t_{n, v}\right) \\
u_{n}\left(t_{n}+\mu h\right)=f(0)+h \sum_{p=0}^{n-1} \sum_{v=1}^{m}\left(\int_{0}^{1} L_{v}(\tau) d \tau\right) w_{p}\left(t_{p, v}\right)+h \sum_{v=1}^{m}\left(\int_{0}^{\mu} L_{v}(\tau) d \tau\right) w_{n}\left(t_{n, v}\right),
\end{gathered}
$$

where $L_{j}(\mu)=\prod_{l \neq j}^{m} \frac{\mu-c_{l}}{c_{j}-c_{l}}$ are the Lagrange polynomials associate with the parameters $c_{j}, j=1, \ldots, m$. By using (2.3), the collocation equation (2.1) may be rewritten as the following nonlinear Volterra integro-differential equation with respect to $w$.

$$
\begin{aligned}
& w_{n}(t)=f(t)+Q\left(t, f(0)+\int_{0}^{t} w(r) d r\right)+\int_{0}^{t} K\left(t, s, f(0)+\int_{0}^{s} w(r) d r, w(s)\right) d s \\
& 0 \leq \tau \leq s \leq t=t_{n, j}, j=1, \ldots, m .
\end{aligned}
$$


Hence, for each $j=1, \ldots, m, n=0, \ldots, N-1, w_{n}\left(t_{n, j}\right)$ satisfies the following nonlinear system,

$$
\begin{aligned}
w_{n}\left(t_{n, j}\right)= & f\left(t_{n, j}\right)+Q\left(t_{n, j}, f(0)+h \sum_{p=0}^{n-1} \int_{0}^{1} w_{p}\left(t_{p}+\tau h\right) d \tau+h \int_{0}^{c_{j}} w_{n}\left(t_{n}+\tau h\right) d \tau\right) \\
& +h \sum_{p=0}^{n-1} \int_{0}^{1} K\left(t_{p, j}, t_{p}+\mu h, u_{p}\left(t_{p}+\mu h\right), w_{p}\left(t_{p}+\mu h\right)\right) d \mu \\
& +h \int_{0}^{c_{j}} K\left(t_{n, j}, t_{n}+\mu h, u_{n}\left(t_{n}+\mu h\right), w_{n}\left(t_{n}+\mu h\right)\right) d \mu,
\end{aligned}
$$

Since the above system is nonlinear, we will use an iterative collocation solution $u^{q} \in S_{m}^{0}\left(I, \Pi_{N}\right), q \in \mathbb{N}$, to approximate the exact solution of (1.1) such that

$$
w_{n}^{q}\left(t_{n}+\mu h\right)=\sum_{v=1}^{m} L_{v}(\mu) w_{n}^{q}\left(t_{n, v}\right)
$$

and

$$
u_{n}^{q}\left(t_{n}+\mu h\right)=f(0)+h \sum_{p=0}^{n-1} \sum_{v=0}^{m}\left(\int_{0}^{1} L_{v}(\tau) d \tau\right) w_{p}^{q}\left(t_{p, v}\right)+h \sum_{v=0}^{m}\left(\int_{0}^{\mu} L_{v}(\tau) d \tau\right) w_{n}^{q}\left(t_{n, v}\right),
$$

where the coefficients $w_{n}^{q}\left(t_{n, j}\right)$ are given by the following formula:

$$
\begin{aligned}
w_{n}^{q}\left(t_{n, j}\right)= & Q\left(t_{n, j}, f(0)+h \sum_{p=0}^{n-1} \int_{0}^{1} w_{p}^{q}\left(t_{p}+\tau h\right) d \tau+h \int_{0}^{c_{j}} w_{n}^{q-1}\left(t_{n}+\tau h\right) d \tau\right) \\
& +f\left(t_{n, j}\right)+h \sum_{p=0}^{n-1} \int_{0}^{1} K\left(t_{p, j}, t_{p}+\mu h, u_{p}^{q}\left(t_{p}+\mu h\right), w_{p}^{q}\left(t_{p}+\mu h\right)\right) d \mu \\
& +h \int_{0}^{c_{j}} K\left(t_{n, j}, t_{n}+\mu h, H_{n}^{q-1}\left(t_{n}+\mu h\right), w_{n}^{q-1}\left(t_{n}+\mu h\right)\right) d \mu
\end{aligned}
$$

where,

$H_{n}^{q}\left(t_{n}+\mu h\right)=f(0)+h \sum_{p=0}^{n-1} \sum_{v=0}^{m}\left(\int_{0}^{1} L_{v}(\tau) d \tau\right) w_{p}^{q}\left(t_{p, v}\right)+h \sum_{v=0}^{m}\left(\int_{0}^{\mu} L_{v}(\tau) d \tau\right) w_{n}^{q-1}\left(t_{n, v}\right)$.

Such that the initial values $w_{n}^{0}\left(t_{n, j}\right)$ belong in a bounded interval $J$.

Remark 2.1. The above formula is explicit and the approximate solution $u^{q}$ is given without needed to solve any algebraic system.

In the next section, we will prove the convergence of the approximate solution $u^{q}$ to the exact solution $x$ of (1.1), moreover, the order of convergence is $m$ for all $q \geq m$. 


\section{Convergence analysis}

In this section, we assume that the functions $Q$ and $K$ satisfy the following Lipschitz conditions: there exist $A_{i} \geq 0 i=0,1,2$ such that

$$
\begin{aligned}
& \left|Q\left(t, x_{1}\right)-Q\left(t, x_{2}\right)\right| \leq A_{0}\left|x_{1}-x_{2}\right|, \\
& \left|K\left(t, s, x_{1}, y_{1}\right)-K\left(t, s, x_{2}, y_{2}\right)\right| \leq A_{1}\left|x_{1}-x_{2}\right|+A_{2}\left|y_{1}-y_{2}\right| .
\end{aligned}
$$

The following lemma will be used in this section.

Lemma 3.1. [3] Let $\left\{k_{j}\right\}_{j=0}^{n}$ be a given non-negative sequence and the sequence $\left\{\varepsilon_{n}\right\}$ satisfies $\varepsilon_{0} \leq p_{0}$ and

$$
\varepsilon_{n} \leq p_{0}+\sum_{i=0}^{n-1} k_{i} \varepsilon_{i}, \quad n \geq 1,
$$

with $p_{0} \geq 0$. Then $\varepsilon_{n}$ can be bounded by

$$
\varepsilon_{n} \leq p_{0} \exp \left(\sum_{j=0}^{n-1} k_{j}\right), \quad n \geq 1 .
$$

The following result gives the existence and the uniqueness of a solution for the nonlinear system (2.4).

Lemma 3.2. For sufficiently small $h$, the nonlinear system (2.4) has a unique solution $u \in S_{m}^{0}\left(I, \Pi_{N}\right)$.

Proof. We will use the induction combined with the Banach fixed point theorem.

(i) On the interval $\sigma_{0}=\left[t_{0}, t_{1}\right]$, the nonlinear system (2.4) becomes

$$
\begin{aligned}
w_{0}\left(t_{0, j}\right)= & f\left(t_{0, j}\right)+Q\left(t_{0, j}, u_{0}+h \sum_{v=1}^{m}\left(\int_{0}^{c_{j}} L_{v}(\tau) d \tau\right) w_{0}\left(t_{0, v}\right)\right) \\
& +h \int_{0}^{c_{j}} K\left(t_{0, j}, t_{0}+\mu h, u_{0}+h \sum_{v=1}^{m}\left(\int_{0}^{\mu} L_{v}(\tau) d \tau\right) w_{0}\left(t_{0, v}\right),\right. \\
& \left.\sum_{v=1}^{m} L_{v}(\mu) w_{0}\left(t_{0, v}\right)\right) d \mu .
\end{aligned}
$$

We consider the operator $\Psi$ defined by:

$$
\begin{aligned}
\Psi: \mathbb{R}^{m} & \longrightarrow \mathbb{R}^{m} \\
x=\left(x_{1}, \ldots, x_{m}\right) & \longmapsto \Psi(x)=\left(\Psi_{1}(x), \ldots, \Psi_{m}(x)\right),
\end{aligned}
$$

such that for $j=1, \ldots, m$, we have

$$
\begin{aligned}
\Psi_{j}(x)= & f\left(t_{0, j}\right)+Q\left(t_{0, j}, u_{0}+h \sum_{v=1}^{m}\left(\int_{0}^{c_{j}} L_{v}(\tau) d \tau\right) x_{v}\right) \\
& +h \int_{0}^{c_{j}} K\left(t_{0, j}, t_{0}+\mu h, u_{0}+h \sum_{v=1}^{m}\left(\int_{0}^{\mu} L_{v}(\tau) d \tau\right) x_{v}, \sum_{v=1}^{m} L_{v}(\mu) x_{v}\right) d \mu .
\end{aligned}
$$


Hence, for all $x, y \in \mathbb{R}^{m}$, we have

$$
\|\Psi(x)-\Psi(y)\|_{\infty} \leq h m b\left(A_{0}+A_{1}+A_{2}\right)\|x-y\|_{\infty},
$$

where $b=\max \left\{\left|L_{v}(\mu)\right|, \mu \in[0,1], v=1, \ldots, m\right\}$.

Since $h m b\left(A_{0}+A_{1}+A_{2}\right)<1$ for sufficiently small $h$, then by Banach fixed point theorem, the nonlinear system (3.1) has a unique solution on the interval $\sigma_{0}$.

(ii) Suppose that $u$ exists and unique on the intervals $\sigma_{i}, i=0, \ldots, n-1$ for $n \geq 1$ and we show that $u$ exists and unique on the interval $\sigma_{n}$.

On the interval $\sigma_{n}$, the nonlinear system (2.4) becomes

$$
\begin{aligned}
w_{n}\left(t_{n, j}\right)= & F\left(t_{n, j}\right)+Q\left(t_{n, j}, G\left(t_{n, j}\right)+h \sum_{v=1}^{m}\left(\int_{0}^{c_{j}} L_{v}(\tau) d \tau\right) w_{n}\left(t_{n, v}\right)\right) \\
& +h \int_{0}^{c_{j}} K\left(t_{n, j}, t_{n}+\mu h, R\left(t_{n, j}\right)+h \sum_{v=1}^{m}\left(\int_{0}^{\mu} L_{v}(\tau) d \tau\right) w_{n}\left(t_{n, v}\right),\right. \\
& \left.\sum_{v=1}^{m} L_{v}(\mu) w_{n}\left(t_{n, v}\right)\right) d \mu
\end{aligned}
$$

where,

$$
\begin{aligned}
& F\left(t_{n, j}\right)=f\left(t_{n, j}\right)+h \sum_{p=0}^{n-1} \int_{0}^{1} K\left(t_{p, j}, t_{p}+\mu h, u_{p}\left(t_{p}+\mu h\right), w_{p}\left(t_{p}+\mu h\right)\right) d \mu . \\
& G\left(t_{n, j}\right)=f(0)+h \sum_{p=0}^{n-1} \sum_{v=1}^{m}\left(\int_{0}^{1} L_{v}(\tau) d \tau\right) w_{p}\left(t_{p, v}\right) .
\end{aligned}
$$

We consider the operator $\Psi$ defined by:

$$
\begin{aligned}
\Psi: \mathbb{R}^{m} & \longrightarrow \mathbb{R}^{m} \\
x=\left(x_{1}, \ldots, x_{m}\right) & \longmapsto \Psi(x)=\left(\Psi_{1}(x), \ldots, \Psi_{m}(x)\right),
\end{aligned}
$$

such that for $j=1, \ldots, m$, we have

$$
\begin{aligned}
\Psi_{j}(x)= & F\left(t_{n, j}\right)+Q\left(t_{n, j}, G\left(t_{n, j}\right)+h \sum_{v=1}^{m}\left(\int_{0}^{c_{j}} L_{v}(\tau) d \tau\right) x_{v}\right) \\
& +h \int_{0}^{c_{j}} K\left(t_{n, j}, t_{n}+\mu h, G\left(t_{n, j}\right)+h \sum_{v=1}^{m}\left(\int_{0}^{\mu} L_{v}(\tau) d \tau\right) x_{v}, \sum_{v=1}^{m} L_{v}(\mu) x_{v}\right) d \mu
\end{aligned}
$$

Hence, for all $x, y \in \mathbb{R}^{m}$, we have

$$
\|\Psi(x)-\Psi(y)\|_{\infty} \leq h m b\left(A_{0}+A_{1}+A_{2}\right)\|x-y\|_{\infty},
$$

Since $h m b\left(A_{0}+A_{1}+A_{2}\right)<1$ for sufficiently small $h$, then by Banach fixed point theorem, the nonlinear system (2.4) has a unique solution $u$ on $\sigma_{n}$.

The following result gives the convergence of the approximate solution $u$ to the exact solution $x$. 
Theorem 3.1. Let $f, Q, K$ be $m$ times continuously differentiable on their respective domains. Then for sufficiently small $h$, the collocation solution u converges to the exact solution $x$, and the resulting error function $e:=x-u$ satisfies:

$$
\left\|e^{v}\right\|_{L^{\infty}(I)} \leq C h^{m}
$$

for $v=0,1$, where $C$ is a finite constant independent of $h$.

Proof. Let $y=x^{\prime}$. It holds that

$$
y_{n}\left(t_{n}+\mu h\right)=\sum_{j=1}^{m} L_{j}(\mu) y_{n}\left(t_{n, j}\right)+\epsilon_{n}(\mu), \epsilon_{n}(\mu)=h^{m} \frac{y^{m}\left(\zeta_{n}(\mu)\right)}{m !} \prod_{j=1}^{m}\left(\mu-c_{j}\right) \text {. }
$$

Hence,

$$
\begin{aligned}
x_{n}\left(t_{n}+\mu h\right)=u_{0} & +h \sum_{p=0}^{n-1} \int_{0}^{1}\left(\sum_{v=1}^{m} L_{v}(\tau) y_{p}\left(t_{p, v}\right)+h^{m} \frac{y^{m}\left(\zeta_{p}(\tau)\right)}{m !} \prod_{j=1}^{m}\left(\tau-c_{j}\right) d \tau\right) \\
& +h \int_{0}^{\mu}\left(\sum_{v=1}^{m} L_{v}(\tau) y_{n}\left(t_{n, v}\right)+h^{m} \frac{y^{m}\left(\zeta_{n}(\tau)\right)}{m !} \prod_{j=1}^{m}\left(\tau-c_{j}\right)\right) d \tau
\end{aligned}
$$

It follows that the errors $\xi=y-w$ and $e=x-u$ have the following representation

$$
\begin{aligned}
\xi_{n}\left(t_{n}+\mu h\right)= & \sum_{j=1}^{m} L_{j}(\mu) \xi_{n}\left(t_{n, j}\right)+\epsilon_{n}(\mu), \epsilon_{n}(\mu)=h^{m} \frac{y^{m}\left(\zeta_{n}(\mu)\right)}{m !} \prod_{j=1}^{m}\left(\mu-c_{j}\right), \\
e\left(t_{n}+\mu h\right)= & h \sum_{p=0}^{n-1} \int_{0}^{1}\left(\sum_{v=1}^{m} L_{v}(\tau) \xi_{p}\left(t_{p, v}\right)+h^{m} \frac{y^{m}\left(\zeta_{p}(\tau)\right)}{m !} \prod_{j=1}^{m}\left(\tau-c_{j}\right)\right) d \tau \\
& +h \int_{0}^{\mu}\left(\sum_{v=1}^{m} L_{v}(\tau) \xi_{n}\left(t_{n, v}\right)+h^{m} \frac{y^{m}\left(\zeta_{n}(\tau)\right)}{m !} \prod_{j=1}^{m}\left(\tau-c_{j}\right)\right) d \tau
\end{aligned}
$$

where $\xi_{n}=\left.\xi\right|_{\sigma_{n}}$ and $e_{n}=\left.e\right|_{\sigma_{n}}$.

On the other hand, from (2.4), we have

$$
\begin{aligned}
\left|\xi_{n}\left(t_{n, j}\right)\right| \leq & h A b \sum_{p=0}^{n} \sum_{v=1}^{m}\left|\xi_{p}\left(t_{p, v}\right)\right| \\
& +h A b \sum_{p=0}^{n-1}\left(h \sum_{i=0}^{p-1} \sum_{v=1}^{m}\left|\xi_{i}\left(t_{i, v}\right)\right|+h \sum_{v=1}^{m}\left|\xi_{p}\left(t_{p, v}\right)\right|+\sum_{v=1}^{m}\left|\xi_{p}\left(t_{p, v}\right)\right|\right) \\
& +h A b\left(h \sum_{p=0}^{n-1} \sum_{v=1}^{m}\left|\xi_{p}\left(t_{p, v}\right)\right|+h \sum_{v=1}^{m}\left|\xi_{n}\left(t_{n, v}\right)\right|+\sum_{v=1}^{m}\left|\xi_{n}\left(t_{n, v}\right)\right|\right)+\alpha h^{m}
\end{aligned}
$$

where $A=\max \left\{A_{i}, i=0,1,2\right\}$ and $\alpha$ is a positive number.

We consider the sequence $\xi_{n}=\max \left\{\left|\xi_{n}\left(t_{n, v}\right)\right|\right.$ for $n=0, \ldots, N-1$. 
Then, from $(3.6), \xi_{n}$ satisfies for $n=0, \ldots, N-1$,

$$
\begin{aligned}
\xi_{n} \leq & A h b m \sum_{p=0}^{n} \xi_{p}+h A b m \sum_{p=0}^{n-1}\left(h \sum_{i=0}^{p-1} \xi_{i}+h \xi_{p}+\xi_{p}\right) \\
& +h A b m\left(h \sum_{p=0}^{n-1} \xi_{p}+h \xi_{n}+\xi_{n}\right)+\alpha h^{m} \\
\leq & h \underbrace{A b m(2+3 T)}_{\alpha_{1}} \sum_{p=0}^{n-1} \xi_{p}+h \underbrace{A b m(T+2)}_{\alpha_{2}} \xi_{n}+\alpha h^{m} .
\end{aligned}
$$

Hence, for $\bar{h}<\frac{1}{\alpha_{2}}$, we have for all $h \in(0, \bar{h}]$

$$
\xi_{n} \leq \frac{\alpha}{1-\bar{h} \alpha_{2}} h^{m}+\frac{\alpha_{1}}{1-\bar{h} \alpha_{2}} h \sum_{p=0}^{n-1} \xi_{p} .
$$

Then, by Lemma 3.1 , for all $n=0, \ldots, N-1$

$$
\xi_{n} \leq \frac{\alpha}{1-\bar{h} \alpha_{2}} h^{m} \exp \left(\frac{T \alpha_{1}}{1-\bar{h} \alpha_{2}}\right) .
$$

Therefore, by using (3.4), we obtain

$$
\begin{aligned}
\|e\| & \leq m b \max \left\{\xi_{n}, n=0, \ldots, N-1\right\}+\beta h^{m} \\
& \leq m b \frac{\alpha}{1-\bar{h} \alpha_{2}} \exp \left(\frac{T \alpha_{1}}{1-\bar{h} \alpha_{2}}\right) h^{m}+\beta h^{m} \\
& \leq \underbrace{\left(m b \frac{\alpha}{1-\bar{h} \alpha_{2}} \exp \left(\frac{T \alpha_{1}}{1-\bar{h} \alpha_{2}}\right)+\beta\right)}_{\alpha_{3}} h^{m},
\end{aligned}
$$

where $\beta$ is a positive number.

Therefore, by using (3.5), we obtain

$$
\|e\| \leq h m b \sum_{p=0}^{n-1} \xi_{p}+h m b \xi_{n}+\gamma h^{m} \leq 2 m b T \alpha_{3} h^{m}+\gamma h^{m},
$$

where $\gamma$ is a positive number,

Thus, the proof is completed by taking $C=\max \left(\alpha_{3}, 2 m b T \alpha_{3}+\gamma\right)$.

The following result gives the convergence of the iterative solution $u^{q}$ to the exacts solution $x$.

Theorem 3.2. Consider the iterative collocation solution $u^{q}$ defined by (2.5), (2.6) and (2.7), then for any initial conditions $\left(u^{\prime}\right)^{0}\left(t_{n, j}\right)=w^{0}\left(t_{n, j}\right) \in J(J$ is a bounded interval), the iterative collocation solution $u^{q}$ converges to the exact solution x. Moreover, the following error estimates hold

$$
\left\|\left(u^{q}\right)^{(v)}-x^{(v)}\right\| \leq C h^{m}+C^{\prime} \beta^{q} h^{q}
$$

for $v=0,1$, where $C, C^{\prime}, \beta$ are finite constants independent of $h$ and $q$. 
Proof. We define the error $\xi^{q}, e^{q}, \varepsilon^{q}$ and $\varsigma^{q}$ by $\xi^{q}(t)=w^{q}(t)-y(t), e^{q}(t)=$ $u^{q}(t)-x(t), \quad \varepsilon^{q}=w^{q}(t)-w(t), \quad \varsigma^{q}=u^{q}(t)-u(t)$ where $u$ is defined by lemma 3.2 .

We have, from (2.4) and (2.7), for all $n=0, \ldots, N-1$ and $j=1, \ldots, m$

$$
\begin{aligned}
\left|\varepsilon_{n}^{q}\left(t_{n, j}\right)\right| \leq & h A b\left(\sum_{p=0}^{n-1} \sum_{v=0}^{m}\left|\varepsilon_{p}^{q}\left(t_{p, v}\right)\right|+\sum_{v=0}^{m}\left|\varepsilon_{n}^{q-1}\left(t_{n, v}\right)\right|\right) \\
& +h A b \sum_{p=0}^{n-1}\left(h \sum_{i=0}^{p-1} \sum_{v=0}^{m}\left|\varepsilon_{i}^{q}\left(t_{i, v}\right)\right|+h \sum_{v=0}^{m}\left|\varepsilon_{p}^{q}\left(t_{p, v}\right)\right|+\sum_{v=0}^{m}\left|\varepsilon_{p}^{q}\left(t_{p, v}\right)\right|\right) \\
& +h A b\left(h \sum_{p=0}^{n-1} \sum_{v=0}^{m}\left|\varepsilon_{p}^{q}\left(t_{p, v}\right)\right|+h \sum_{v=0}^{m}\left|\varepsilon_{n}^{q-1}\left(t_{n, v}\right)\right|+\sum_{v=0}^{m}\left|\varepsilon_{n}^{q-1}\left(t_{n, v}\right)\right|\right),
\end{aligned}
$$

Now, for each fixed $q \geq 1$, we consider the sequence $\varepsilon_{n}^{q}=\max \left\{\left|\varepsilon_{n}^{q}\left(t_{n, v}\right)\right| v=\right.$ $1 \ldots . m\}$. It follows, from (3.8), that for $n=0, \ldots, N-1$

$$
\begin{aligned}
& \varepsilon_{n}^{q} \leq h \operatorname{Abm}\left(\sum_{p=0}^{n-1} \varepsilon_{p}^{q}+\varepsilon_{n}^{q-1}\right)+h A b m \sum_{p=0}^{n-1}\left(h \sum_{i=0}^{p-1} \varepsilon_{i}^{q}+h \varepsilon_{p}^{q}+\varepsilon_{p}^{q}\right) \\
& +h \operatorname{Abm}\left(h \sum_{p=0}^{n-1} \varepsilon_{p}^{q}+h \varepsilon_{n}^{q-1}+\varepsilon_{n}^{q-1}\right) \\
& \leq h \underbrace{\operatorname{Abm}(2+3 T)}_{\alpha_{1}} \sum_{p=0}^{n-1} \varepsilon_{p}^{q}+h \underbrace{A b m(2+T)}_{\alpha_{2}} \varepsilon_{n}^{q-1},
\end{aligned}
$$

We consider the sequence $\eta^{q}=\max \left\{\varepsilon_{n}^{q}, n=0, \ldots, N-1\right\}$ for $q \geq 1$.

Then, from (3.9), we obtain

$$
\varepsilon_{n}^{q} \leq \alpha_{1} h \sum_{p=0}^{n-1} \varepsilon_{p}^{q}+\alpha_{2} h \eta^{q-1} .
$$

Hence, by Lemma 3.1, for all $n=0, \ldots, N-1$

$$
\eta^{q} \leq \underbrace{\alpha_{2} \exp \left(\alpha_{1} T\right)}_{\beta} h \eta^{q-1} \leq \beta^{2} h^{2} \eta^{q-2} \leq \ldots \leq \beta^{q} h^{q} \eta^{0} .
$$

Since, $w^{0}\left(t_{n, j}\right) \in J$ (bounded interval) and $w$ is bounded by Lemma 3.2, then there exists $\delta>0$ such that $\eta^{0}<\delta$, which implies that, for all $q \geq 1$

$$
\eta^{q} \leq \delta \beta^{q} h^{q}
$$

Therefore, by using (2.2) and (2.5), we obtain

$$
\left\|\varepsilon^{q}\right\| \leq m b \eta^{q} \leq \underbrace{m b \delta}_{d} \beta^{q} h^{q}
$$

Hence, by Theorem (3.1), we deduce that

$$
\left\|\xi^{q}\right\| \leq\left\|\varepsilon^{q}\right\|+\|w-y\| \leq d \beta^{q} h^{q}+C h^{m} .
$$


On the other hand, from (2.3) and (2.6), we have

$$
\left\|\varsigma^{q}\right\| \leq 2 T m b\left\|\varepsilon^{q}\right\| \leq 2 T m b d \beta^{q} h^{q} .
$$

Finally, by using Theorem (3.1), we deduce that

$$
\left\|e^{q}\right\| \leq\left\|\varsigma^{q}\right\|+\|u-x\| \leq \underbrace{2 \operatorname{Tmbd}}_{d^{\prime}} \beta^{q} h^{q}+C h^{m} .
$$

Thus, the proof is completed by taking $C^{\prime}=\max \left(d, d^{\prime}\right)$.

\section{Numerical Examples}

In order to test the applicability of the presented method, we consider the following examples with $T=1$. These examples have been solved with various values of $N, m$ and $q=m$. We used the collocation parameters $c_{i}=\frac{i}{m+1}, i=$ $1, \ldots, m$. In each example, we calculate the error between $x$ and the iterative collocation solution $u^{m}$.

The absolute errors at the particular points are given to compare our solutions with the solutions obtained by $[16,18]$.

The results in these examples confirm the theoretical results; moreover, the results obtained by the present method is very superior to that obtained by the methods in $[16,18]$.

Example 4.1. Consider the nonlinear Volterra integro-differential equation given by

$$
x^{\prime}(t)=f(t)+\int_{0}^{t} \cos \left(t+s+x(s)+x^{\prime}(s)\right)+\frac{1}{1+x^{2}(s)} d s, t \in[0,1] .
$$

with $f$ is chosen so that the exact solution is $x(t)=2 t+5$. The absolute errors for $(N, m) \in\{(2,3),(4,3),(4,4),(6,4)\}$ at $t=0,0.1, \ldots, 1$ are presented in Table 1. From the Table 1, we note that the absolute error reduces as $N$ or $m$ increases.

TABLE 1. Absolute errors for Example 4.1

\begin{tabular}{|l|l|l|l|l|}
\hline$t$ & $\begin{array}{l}N=2 \\
m=3\end{array}$ & $\begin{array}{l}N=4 \\
m=3\end{array}$ & $\begin{array}{l}N=4 \\
m=4\end{array}$ & $\begin{array}{l}N=6 \\
m=4\end{array}$ \\
\hline 0 & 0.0 & 0.0 & 0.0 & 0.0 \\
\hline 0.1 & $2.03 \mathrm{E}-4$ & $1.48 \mathrm{E}-5$ & $4.40 \mathrm{E}-7$ & $4.99 \mathrm{E}-8$ \\
\hline 0.2 & $2.37 \mathrm{E}-4$ & $1.73 \mathrm{E}-5$ & $1.36 \mathrm{E}-6$ & $2.90 \mathrm{E}-7$ \\
\hline 0.3 & $2.23 \mathrm{E}-4$ & $4.94 \mathrm{E}-7$ & $1.43 \mathrm{E}-6$ & $2.27 \mathrm{E}-7$ \\
\hline 0.4 & $2.88 \mathrm{E}-4$ & $7.86 \mathrm{E}-6$ & $1.21 \mathrm{E}-6$ & $9.56 \mathrm{E}-7$ \\
\hline 0.5 & $5.53 \mathrm{E}-4$ & $6.69 \mathrm{E}-5$ & $8.55 \mathrm{E}-6$ & $9.58 \mathrm{E}-7$ \\
\hline 0.6 & $1.07 \mathrm{E}-3$ & $6.17 \mathrm{E}-5$ & $7.95 \mathrm{E}-6$ & $8.30 \mathrm{E}-7$ \\
\hline 0.7 & $1.12 \mathrm{E}-3$ & $5.43 \mathrm{E}-5$ & $7.46 \mathrm{E}-6$ & $7.60 \mathrm{E}-7$ \\
\hline 0.8 & $1.14 \mathrm{E}-3$ & $6.04 \mathrm{E}-5$ & $7.24 \mathrm{E}-6$ & $6.97 \mathrm{E}-7$ \\
\hline 0.9 & $1.52 \mathrm{E}-3$ & $6.90 \mathrm{E}-5$ & $6.36 \mathrm{E}-6$ & $3.84 \mathrm{E}-7$ \\
\hline 1 & $2.71 \mathrm{E}-3$ & $1.38 \mathrm{E}-4$ & $6.64 \mathrm{E}-7$ & $4.33 \mathrm{E}-7$ \\
\hline
\end{tabular}


Example 4.2. Consider the nonlinear Volterra integro-differential equation given by

$$
x^{\prime}(t)=f(t)+\int_{0}^{t} \frac{\cos (t)}{1+t+\left(x^{\prime}(s)\right)^{2}}+\frac{t \sin (s)}{2+x^{2}(s)} d s, t \in[0,1] .
$$

with $f$ is chosen so that the exact solution is $x(t)=3 \cos (t)+1$. The absolute errors for $(N, m) \in\{(2,3),(4,3),(4,4),(6,4)\}$ at $t=0,0.1, \ldots, 1$ are presented in Table 2. From the Table 2, we note that the absolute error reduces as $N$ or $m$ increases.

TABLE 2. Absolute errors for Example 4.2

\begin{tabular}{|l|l|l|l|l|}
\hline$t$ & $\begin{array}{l}N=2 \\
m=3\end{array}$ & $\begin{array}{l}N=4 \\
m=3\end{array}$ & $\begin{array}{l}N=4 \\
m=4\end{array}$ & $\begin{array}{l}N=6 \\
m=4\end{array}$ \\
\hline 0 & 0.0 & 0.0 & 0.0 & 0.0 \\
\hline 0.1 & $6.87 \mathrm{E}-4$ & $5.08 \mathrm{E}-5$ & $1.33 \mathrm{E}-7$ & $1.13 \mathrm{E}-8$ \\
\hline 0.2 & $8.11 \mathrm{E}-4$ & $5.17 \mathrm{E}-5$ & $1.36 \mathrm{E}-7$ & $4.96 \mathrm{E}-8$ \\
\hline 0.3 & $8.13 \mathrm{E}-4$ & $8.67 \mathrm{E}-5$ & $5.64 \mathrm{E}-7$ & $6.11 \mathrm{E}-8$ \\
\hline 0.4 & $8.41 \mathrm{E}-4$ & $9.49 \mathrm{E}-5$ & $6.16 \mathrm{E}-7$ & $1.38 \mathrm{E}-7$ \\
\hline 0.5 & $7.58 \mathrm{E}-4$ & $9.41 \mathrm{E}-5$ & $7.46 \mathrm{E}-7$ & $1.61 \mathrm{E}-7$ \\
\hline 0.6 & $1.28 \mathrm{E}-3$ & $1.37 \mathrm{E}-4$ & $1.43 \mathrm{E}-6$ & $2.44 \mathrm{E}-7$ \\
\hline 0.7 & $1.38 \mathrm{E}-3$ & $1.40 \mathrm{E}-4$ & $1.46 \mathrm{E}-6$ & $3.58 \mathrm{E}-7$ \\
\hline 0.8 & $1.39 \mathrm{E}-3$ & $1.65 \mathrm{E}-4$ & $2.38 \mathrm{E}-6$ & $3.69 \mathrm{E}-7$ \\
\hline 0.9 & $1.41 \mathrm{E}-3$ & $1.71 \mathrm{E}-4$ & $2.47 \mathrm{E}-6$ & $5.06 \mathrm{E}-7$ \\
\hline 1 & $1.35 \mathrm{E}-3$ & $1.69 \mathrm{E}-4$ & $2.61 \mathrm{E}-6$ & $5.13 \mathrm{E}-7$ \\
\hline
\end{tabular}

Example 4.3. Consider the nonlinear Volterra integro-differential equation given by

$$
x^{\prime}(t)=f(t)+\int_{0}^{t}\left(t s \arctan \left(s+x(s)+x^{\prime}(s)\right)+\cos (t-s+x(s))\right) d s, t \in[0,1] .
$$

with $f$ is chosen so that the exact solution is $x(t)=2 t+1$. The absolute errors for $(N, m) \in\{(2,2),(2,3),(4,3),(4,4)\}$ at $t=0,0.1, \ldots, 1$ are presented in Table 3 . From the Table 3, we note that the absolute error reduces as $N$ or $m$ increases.

Example 4.4. ([16, 18]) Consider the linear Volterra integro-differential equation given by

$$
x^{\prime}(t)=1-\int_{0}^{t} x(s) d s, t \in[0,1] .
$$

with the initial conditions $x(0)=0$ and the exact solution $x(t)=\sin (t)$. Here, $f(t)=1, g(t)=0, K(t, s)=-1$.

The absolute errors for $N=6,10$ and $m=q=5$ at $t=0,0.1, \ldots, 1$ are displayed in Table 4.

The numerical results of the absolute error function obtained by the present method are compared in Table 4 with the absolute error function of the Taylor method given in [16] and Bessel method [18] for an approximate polynomial solutions of degree 5 . 
TABLE 3. Absolute errors for Example 4.3

\begin{tabular}{|l|l|l|l|l|}
\hline$t$ & $\begin{array}{l}N=2 \\
m=2\end{array}$ & $\begin{array}{l}N=2 \\
m=3\end{array}$ & $\begin{array}{l}N=4 \\
m=3\end{array}$ & $\begin{array}{l}N=4 \\
m=4\end{array}$ \\
\hline 0 & 0.0 & 0.0 & 0.0 & 0.0 \\
\hline 0.1 & $1.26 \mathrm{E}-4$ & $5.86 \mathrm{E}-7$ & $1.40 \mathrm{E}-8$ & $3.03 \mathrm{E}-9$ \\
\hline 0.2 & $2.47 \mathrm{E}-4$ & $1.58 \mathrm{E}-6$ & $5.66 \mathrm{E}-8$ & $8.11 \mathrm{E}-9$ \\
\hline 0.3 & $3.61 \mathrm{E}-4$ & $3.42 \mathrm{E}-6$ & $1.00 \mathrm{E}-7$ & $1.49 \mathrm{E}-9$ \\
\hline 0.4 & $4.70 \mathrm{E}-4$ & $6.53 \mathrm{E}-6$ & $1.13 \mathrm{E}-7$ & $6.14 \mathrm{E}-9$ \\
\hline 0.5 & $5.73 \mathrm{E}-4$ & $1.13 \mathrm{E}-5$ & $1.47 \mathrm{E}-7$ & $2.00 \mathrm{E}-9$ \\
\hline 0.6 & $5.66 \mathrm{E}-4$ & $1.13 \mathrm{E}-5$ & $1.43 \mathrm{E}-7$ & $2.16 \mathrm{E}-9$ \\
\hline 0.7 & $5.63 \mathrm{E}-4$ & $1.11 \mathrm{E}-5$ & $1.41 \mathrm{E}-7$ & $1.01 \mathrm{E}-8$ \\
\hline 0.8 & $5.66 \mathrm{E}-4$ & $1.06 \mathrm{E}-5$ & $1.31 \mathrm{E}-7$ & $4.96 \mathrm{E}-10$ \\
\hline 0.9 & $5.73 \mathrm{E}-4$ & $1.01 \mathrm{E}-5$ & $1.26 \mathrm{E}-7$ & $1.76 \mathrm{E}-10$ \\
\hline 1 & $5.84 \mathrm{E}-4$ & $9.46 \mathrm{E}-6$ & $1.22 \mathrm{E}-7$ & $2.00 \mathrm{E}-9$ \\
\hline
\end{tabular}

TABle 4. Comparison of the absolute errors of Example 4.4

\begin{tabular}{|l|l|l|l|l|}
\hline$t$ & $\begin{array}{l}\text { Taylor method } \\
{[16]}\end{array}$ & $\begin{array}{l}\text { Bessel method } \\
{[18]}\end{array}$ & $\begin{array}{l}\text { Present method } \\
N=6\end{array}$ & $\begin{array}{l}\text { Present method } \\
N=10\end{array}$ \\
\hline 0.0 & 0.0 & 0.0 & 0.0 & 0.0 \\
\hline 0.1 & $2.00 \mathrm{E}-11$ & $2.49 \mathrm{E}-7$ & $1.58 \mathrm{E}-9$ & $1.26 \mathrm{E}-10$ \\
\hline 0.2 & $2.50 \mathrm{E}-9$ & $4.02 \mathrm{E}-7$ & $5.45 \mathrm{E}-10$ & $9.50 \mathrm{E}-11$ \\
\hline 0.3 & $4.33 \mathrm{E}-8$ & $3.00 \mathrm{E}-7$ & $2.71 \mathrm{E}-9$ & $2.61 \mathrm{E}-10$ \\
\hline 0.4 & $3.24 \mathrm{E}-7$ & $2.05 \mathrm{E}-7$ & $8.90 \mathrm{E}-10$ & $1.08 \mathrm{E}-10$ \\
\hline 0.5 & $1.54 \mathrm{E}-6$ & $2.83 \mathrm{E}-7$ & $5.60 \mathrm{E}-9$ & $2.04 \mathrm{E}-10$ \\
\hline 0.6 & $5.52 \mathrm{E}-6$ & $3.75 \mathrm{E}-7$ & $7.20 \mathrm{E}-9$ & $4.96 \mathrm{E}-12$ \\
\hline 0.7 & $1.62 \mathrm{E}-5$ & $1.65 \mathrm{E}-7$ & $1.19 \mathrm{E}-9$ & $4.62 \mathrm{E}-10$ \\
\hline 0.8 & $4.12 \mathrm{E}-5$ & $1.81 \mathrm{E}-7$ & $1.36 \mathrm{E}-10$ & $5.00 \mathrm{E}-10$ \\
\hline 0.9 & $9.38 \mathrm{E}-5$ & $1.18 \mathrm{E}-6$ & $1.27 \mathrm{E}-9$ & $1.72 \mathrm{E}-10$ \\
\hline 1.0 & $1.95 \mathrm{E}-4$ & $9.66 \mathrm{E}-6$ & $7.85 \mathrm{E}-9$ & $9.92 \mathrm{E}-10$ \\
\hline
\end{tabular}

\section{Conclusion}

In this paper, we have used an iterative collocation method based on the Lagrange polynomials to construct continuous iterative numerical solutions for nonlinear Volterra integro-differential equations (1.1) in the spline space $S_{m}^{(0)}\left(\Pi_{N}\right)$. The main advantages of this method that, is easy to implement, has high order of convergence and the coefficients of the approximation solution are determined by using iterative formulas without the need to solve any system of algebraic equations. Numerical examples showing that the method is convergent with a good accuracy and the comparison of the results obtained by the present method with the other methods reveals that the method is very effective and convenient. 


\section{Acknowledgements}

A. Bellour and K. Rouibah acknowledge support from Directorate General for Scientific Research and Technological Development, Algeria.

\section{References}

[1] K.E. Atkinson, The Numerical Solution of Integral Equations of the Second Kind. Cambridge University Press, Cambridge, 1997.

[2] H. Brunner, iterated collocation methods for volterra integral equations with delay arguments, Math. Comp. 62 (1994), 581-599.

[3] H. Brunner, Collocation methods for Volterra integral and related functional differential equations, Cambridge university press, Cambridge, 2004.

[4] H. Brunner, Runge-Kutta convolution quadrature methods with convergence and stability analysis for nonlinear singular fractional integro-differential equations. Commun. Nonlinear. Sci. Numer. Simul. 84 (2020), 105-132.

[5] A. Cardone and D. Conte, Multistep collocation methods for Volterra integrodifferential equations, Appl. Math. Comput. 221 (2013), 770-785.

[6] S. Fazeli and G. Hojjati, Numerical solution of Volterra integro-differential equations by superimplicit multistep collocation methods. Numer. Algorithms. 68 (2015), 741768 .

[7] L. Hacia, Iterative-Collocation Method for Integral Equations of Heat Conduction Problems. In: Boyanov T., Dimova S., Georgiev K., Nikolov G. (eds) Numerical Methods and Applications. NMA 2006. Lecture Notes in Computer Science, vol 4310. Springer, Berlin, Heidelberg, 2007.

[8] R. Kress, Linear Integral Equations. Springer-Verlag, NewYork, 1999.

[9] P.K. Kythe and P. Puri, Computational methods for linear integral equations. Birkhauser-Verlag, Springer, Boston, 2002.

[10] H. Laib, A. Bellour and M. Bousselsal, Numerical solution of high-order linear Volterra integro-differential equations by using Taylor collocation method. Int. J. Comput. Math., 96 (2019), no. 5, 1066-1085.

[11] H. Liang, H. Brunner, On the convergence of collocation solutions in continuous piecewise polynomial spaces for Volterra integral equations. BIT Numer. Math. 56 (2016), 1339-1367.

[12] P. Markowich and M. Renardy, A nonlinear Volterra integro-differential equation describing the stretching of polymeric liquids. SIAM J. Math. Anal. 14 (1983), 6697.

[13] N. Rohaninasab, K. Maleknejad and R. Ezzati, Numerical solution of high-order Volterra integro-differential equations by using Legendre collocation method, Appl. Math. Comput. 328 (2018), 171-188.

[14] K. Rouibah, A. Bellour, P. Lima, and E. Rawashdeh, Iterative continuous collocation method for solving nonlinear volterra integral equations, Kragujev. J. Math. 46 (2022), no. 4, 635-648.

[15] S.H. Wang, J.H. He, Variational iteration method for solving integro-differential equations, Phys. Lett. A. 367 (2007), 188-191.

[16] S. Yalçinbaşa, M. Sezer, The approximate solution of high-order linear VolterraFredholm integro-differential equations in terms of Taylor polynomials, Appl. Math. Comput. 112 (2000), 291-308.

[17] S. Yüzbaşi, M. Sezer and B. Kemanci, Numerical solution of integro-differential equations and application of a population model with an improved Legendre method, Appl. Math. Model. 37 (2013), 2086-2101. 
[18] Ş. Yüzbaşi, N. Şahin, M. Sezer, Bessel polynomial solutions of high-order linear Volterra integro-differential equations, Comput. Math. with Appl., 62 (2011), 19401956.

Azzeddine Bellour
Laboratory of Applied Mathematics and Didactics,
Ecole Normale Supérieure de Constantine, Algeria
E-mail address: bellourazze123@yahoo.com
Khaoula Rouibah
Centre universitaire de Mila, Algeria.
E-mail address: r.khoula@centre-univ-mila.dz
Received: October 21, 2020; Accepted: January 11, 2021 